\title{
Effects of dissipation on disordered quantum spin models
}

\author{
L. F. Cugliandolo ${ }^{1,2}$, D. R. Grempel ${ }^{3}$, G. Lozano ${ }^{4,5}$ and H. Lozza ${ }^{5}$ \\ ${ }^{1}$ Laboratoire de Physique Théorique de l'École Normale Supérieure, \\ 24 rue Lhomond, 75231 Paris Cedex 05, France \\ ${ }^{2}$ Laboratoire de Physique Théorique et Hautes Énergies, Jussieu, \\ 1er étage, Tour 16, 4 Place Jussieu, 75252 Paris Cedex 05, France \\ ${ }^{3}$ CEA-Saclay/SPCSI, 91191 Gif-sur-Yvette CEDEX, France \\ ${ }^{4}$ Department of Mathematics, Imperial College London, \\ 180 Queen's Gate, London SW7 2BZ, United Kingdom \\ ${ }^{5}$ Departamento de Física, FCEyN, Universidad de Buenos Aires, \\ Pabellón I, Ciudad Universitaria, 1428 Buenos Aires, Argentina
}

September 13, 2018

\begin{abstract}
We study the effects of the coupling to an Ohmic quantum reservoir on the static and dynamical properties of a family of disordered $S U(2)$ spin models in a transverse magnetic field using a method of direct spin summation. The tendency to form a glassy phase increases with the strength of the coupling of the system to the environment. We study the influence of the environment on the features of the phase diagram of the various models as well as the stability of the possible phases.

\section{Introduction}

The coupling of quantum two-level systems (TLS) to a dissipative environment has decisive effects on their dynamical properties. The case of dilute systems, in which interactions between the TLS can be neglected, has been extensively investigated in the literature [1, 2] and is now well understood. The physics that emerges when interactions between the TLS may not be neglected has received much less attention.
\end{abstract}


In this paper we study the effects of a dissipative environment on the equilibrium and dynamical properties of quantum glassy systems. While spin-glass phases in which quantum fluctuations play an important role were found in a number of experimental systems [3, 4, [5], the importance of interactions on the properties of tunneling defects in structural glasses was recently demonstrated [6].

Mean-field [7] and finite dimensional [8] quantum spin-glass models have been studied in detail in the last few years. The interest in the effects of the coupling to the environment on the properties of quantum glassy systems is more recent. The quantum spherical $p$-spin glass model [9, 10, 11, the $S U(N)$ random Heisenberg model in the limit $N \rightarrow \infty$ [12], and the quantum random walk problem [13] were studied in detail. In these cases the relevant degrees of freedom are continuous which makes analytical treatments possible. Here we analyze the more realistic case of quantum $S U(2)$ spins with infinite range interactions between $p$-uplets of spins coupled to a bath of harmonic oscillators. For $p=2$ this model reduces a model for metallic spin-glasses studied previously [14]. For $p>3$ the model exhibits richer behavior [15, 16, 17, 18, including the possibility of having first order transition lines.

We use a method of direct spin summation (DSS) first used in the context of disordered spin models by Goldschmidt and Lai [19. In this method the disorderaveraged free-energy density is computed using the replica method to average over the random quenched interactions. Next, a Trotter decomposition is performed in order to express the partition function of the resulting single-site self-consistent problem as a sum over different contributions, each coming from a possible spin history $\sigma(\tau)= \pm 1$. The continuous imaginary-time variable $0 \leq \tau \leq \beta \hbar$ is discretized on a grid $\tau_{t}=t \beta \hbar / M, t=0, \cdots, M-1$, and the partition function is computed by numerically performing the exact sum over the $2^{M}$ possible discrete spin histories, $\sigma_{t} \equiv \sigma\left(\tau_{t}\right)= \pm 1$. Physical results are obtained repeating this procedure for various values of $M$ and extrapolating to $M \rightarrow \infty$.

We find that the coupling to the enviromement favors the appearance of the spin-glass phase reducing the strength of the quantum fluctuations that tend to destabilize it. For $p=2$ the phase transition is always second order. For $p \geq 3$ there exists a tricritial temperature $T^{\star}$ below which quantum fluctuations drive the transition first order. $T^{\star}$ decreases with the strength of the coupling to the bath. For $p \geq 3$ a dynamic transition precedes the equilibrium phase transition. The coupling to the bath also stabilizes the dynamic glassy phase. 
The organization of the paper is as follows. In Sect. 2] we introduce the coupled $p$-spin-bath model and the formalism that we use to solve it. In Sect. 3 we discuss the numerical method and present our results. Sect. 4 contains our conclusions.

\section{The model}

We are interested in disordered $S U(2)$ spin models in a transverse field described by Hamiltonians of the type

$$
H_{s}=H_{L}-\Gamma \sum_{i=1}^{N} \hat{\sigma}_{i}^{x}
$$

with

$$
H_{L}=-\sum_{i_{1}<\cdots<i_{p}}^{N} J_{i_{1}, \ldots, i_{p}} \hat{\sigma}_{i_{1}}^{z} \cdots \hat{\sigma}_{i_{p}}^{z} .
$$

Here, $\hat{\sigma}^{x}, \hat{\sigma}^{y}, \hat{\sigma}^{z}$ are the standard Pauli matrices, $J_{i_{1} \cdots i_{p}}$ denotes a quenched random exchange between $p$ spins, $\Gamma$ is the transverse field introducing quantum fluctuations and $N$ is the total number of spins. The sum runs over all possible $p$-uplets of spins. The model is then fully-connected and mean-field in character. It is completely determined once the order $p$ and the precise distribution $\mathcal{P}[J]$ of random interactions are chosen. We consider the case in which the random independent variables $J_{i_{1}, \ldots, i_{p}}$ are Gaussian with zero mean and variance $p ! J^{2} / 2 N^{p-1}$. The scaling of the variance with $N$ is chosen so as to ensure a good thermodynamic limit.

We study the thermodynamics and some aspects of the non-equilibrium dynamics of the quantum spin model coupled to a quantum environment assumed to be in thermal equilibrium. We model the coupling to the environment by assuming that each spin in the system is coupled to its own set of $\tilde{N} / N$ independent harmonic oscillators with $\tilde{N}$ the total number of them. The bosonic Hamiltonian for the isolated reservoir is

$$
H_{b}=\sum_{l=1}^{\tilde{N}} \frac{1}{2 m_{l}} \hat{p}_{l}^{2}+\sum_{l=1}^{\tilde{N}} \frac{1}{2} m_{l} \omega_{l}^{2} \hat{x}_{l}^{2} .
$$

The coordinates $\hat{x}_{l}$ and the momenta $\hat{p}_{l}$ satisfy canonical commutation relations. For simplicity we consider a bilinear coupling,

$$
H_{s b}=-\sum_{i=1}^{N} \hat{\sigma}_{i}^{z} \sum_{l=1}^{\tilde{N}} c_{i l} \hat{x}_{l},
$$


that involves only the oscillator coordinates. The Hamiltonian for the coupled system is then given by

$$
H=H_{s}+H_{b}+H_{s b}+H_{c t}
$$

where we added a counter-term,

$$
H_{c t}=\sum_{l=1}^{\tilde{N}} \frac{1}{2 m_{l} \omega_{l}^{2}}\left(\sum_{i=1}^{N} c_{i l} \hat{\sigma}_{i}^{z}\right)^{2},
$$

whose effect is to eliminate a possible mass-normalization induced by the coupling to the bath [2].

The partition function of the combined system for a particular realization of the bonds

$$
Z=\operatorname{Tr}\left[e^{-\beta H}\right]
$$

involves a sum over all states of the system and of the bath. The trace over the variables of the bath can be performed explicitly using standard techniques [2, 20, 21. The result of tracing out these variables can be expressed in terms of the spectral function of the bath,

$$
I_{i j}(\omega) \equiv \frac{\pi}{2} \sum_{l=1}^{\tilde{N}} \frac{c_{i l} c_{j l}}{m_{l} \omega_{l}} \delta\left(\omega-\omega_{l}\right)=\delta_{i j} I(\omega) .
$$

We chose to study the effect of an Ohmic bath parameterized as

$$
I(\omega)=2 \eta \hbar \omega \Theta\left(\omega_{\max }-\omega\right)
$$

with $\eta$ the friction constant, $\omega_{\max }$ an ultra-violet cut-off and $\Theta(x)$ the Heaviside theta-function.

This problem can be mapped onto a classical Ising spin system using the TotterSuzuki formalism [15, 19, 22]. This amounts to writing the path-integral for the partition function as a sum over spin and oscillator variables evaluated on a discrete imaginary-time grid, $\tau_{t}=\beta \hbar / M t$, labeled by the index $t=0, \ldots, M-1$. Periodic boundary conditions on the discrete time-axis are imposed due to the fact that the evaluation of the partition function involves a trace. To recover the correct representation of the trace the limit $M \rightarrow \infty$ should be ultimately taken. The finite $M$ expression yields a sequence of $M$-approximants to the asymptotic $M \rightarrow \infty$ formula. 
The $M$-th approximant of the "reduced" partition function obtained after integrating out the bath reads

$$
\begin{aligned}
& Z=\operatorname{Tr}_{\left\{\sigma_{i}^{t}\right\}} \exp \left[\frac{\beta}{M} \sum_{t=0}^{M-1} \sum_{i_{1}<\cdots<i_{p}}^{N} J_{i_{1} \cdots i_{p}} \sigma_{i_{1}}^{t} \cdots \sigma_{i_{p}}^{t}+\sum_{t=0}^{M-1} \sum_{i=1}^{N}\left(A+B \sigma_{i}^{t} \sigma_{i}^{(t+1)}\right)\right. \\
&\left.-\sum_{t, t^{\prime}=0}^{M-1} \sum_{i=1}^{N}\left(1-\sigma_{i}^{t} \sigma_{i}^{t^{\prime}}\right) \mathcal{C}_{\left(t-t^{\prime}\right)}\right]
\end{aligned}
$$

where

$$
\begin{aligned}
A & =\frac{1}{2} \ln \left[\sinh \left(\frac{\beta \Gamma}{M}\right) \cosh \left(\frac{\beta \Gamma}{M}\right)\right] \\
B & =\frac{1}{2} \ln \left[\operatorname{coth}\left(\frac{\beta \Gamma}{M}\right)\right] \\
\mathcal{C}_{\left(t-t^{\prime}\right)} & =\frac{2 \eta}{\pi \hbar} \int_{0}^{\omega_{\max }} d \omega \frac{\cosh \left(\omega \beta \hbar\left(\left(t-t^{\prime}\right) / M-1 / 2\right)\right) \sinh ^{2}(\omega \beta \hbar / 2 M)}{\omega \sinh (\omega \beta \hbar / 2)}
\end{aligned}
$$

The trace represents the sum over all $2^{N \times M}$ distinct classical Ising spin configurations, $\sigma_{i}^{t}= \pm 1$, for each spin, $i=1, \ldots, N$, evaluated at each time-slice, $t=0, \ldots, M-1$.

The disordered averaged free-energy is calculated using the replica trick [23]

$$
\beta \bar{F}=-\overline{\ln Z}=-\lim _{n \rightarrow 0} \frac{\overline{Z^{n}}-1}{n} .
$$

After some standard manipulations, and up to some irrelevant factors, we obtain

$$
\overline{Z^{n}}=\prod_{a, b=1}^{n} \prod_{t, t^{\prime}=0}^{M-1} \int \mathcal{D} Q^{a t b t^{\prime}} \mathcal{D} \Lambda^{a t b t^{\prime}} \exp (-N P(\Lambda, Q))
$$

with

$$
\begin{gathered}
P(\Lambda, Q)=\sum_{a, b=1}^{n} \sum_{t, t^{\prime}=0}^{M-1}\left(\frac{\Lambda^{a t b t^{\prime}}}{M^{2}} Q^{a t b t^{\prime}}-\frac{\beta^{2} J^{2}}{4 M^{2}}\left(Q^{a t b t^{\prime}}\right)^{\bullet p}+\mathcal{C}_{\left(t-t^{\prime}\right)} \delta^{a b}\left(1-Q^{a t b t^{\prime}}\right)\right) \\
\quad-\ln \operatorname{Tr}_{\left\{\sigma^{a t}\right\}} e^{H_{e f f}} \\
H_{\text {eff }}=\sum_{a, b=1}^{n} \sum_{t, t^{\prime}=0}^{M-1} \frac{\Lambda^{a t b t^{\prime}}}{M^{2}} \sigma^{a t} \sigma^{b t^{\prime}}+\sum_{a=1}^{n} \sum_{t=0}^{M-1}\left(A+B \sigma^{a t} \sigma^{a(t+1)}\right)
\end{gathered}
$$

where the bullet is used to distinguish the ordinary power from the matrix power. In the thermodynamic limit, $N \rightarrow \infty$, the integrals in $\bar{Z}^{n}$ can be evaluated with the 
saddle point method at the expense of exchanging the $N \rightarrow \infty$ and $n \rightarrow 0$ limits. The disordered-averaged free-energy per spin is then

$$
\beta \bar{f}=-\lim _{n \rightarrow 0} \frac{P\left[\Lambda_{0}, Q_{0}\right]}{n}
$$

where $\Lambda_{0}$ and $Q_{0}$ are such that,

$$
\left.\frac{\delta P(Q, \Lambda)}{\delta Q}\right|_{Q_{0}, \Lambda_{0}}=0,\left.\quad \frac{\delta P(Q, \Lambda)}{\delta \Lambda}\right|_{Q_{0}, \Lambda_{0}}=0
$$

Hereafter we omit subscripts in the saddle-point values $Q_{0}$ and $\Lambda_{0}$. The disorderaveraged entropy per spin is easily obtained from the disorder-averaged free-energy density and reads

$$
\begin{aligned}
-\frac{\bar{s}}{k_{B}}= & \beta \bar{f}+\frac{1}{n} \sum_{a, b=1}^{n} \sum_{t, t^{\prime}=0}^{M-1}\left[\frac{\beta^{2} J^{2}}{2 M^{2}}\left(Q^{a t b t^{\prime}}\right)^{\bullet p}-\delta^{a b}\left(1-Q^{a t b t^{\prime}}\right) \frac{\partial \mathcal{C}_{\left(t-t^{\prime}\right)}}{\partial \beta}\right] \\
& +\frac{\beta \Gamma}{\sinh (2 \beta \Gamma / M)}\left[\cosh (2 \beta \Gamma / M)-\frac{1}{n} \sum_{a=1}^{n} Q^{a(t+1) a t}\right] .
\end{aligned}
$$

Another physical observable of interest is the magnetic susceptibility,

$$
\chi=\left.\frac{\partial \mathcal{M}}{\partial h}\right|_{h=0}
$$

where $\mathcal{M}=N^{-1} \sum_{i=1}^{N} \overline{\left\langle\sigma_{i}^{z}\right\rangle}$ is the total disorder-averaged magnetization and $h$ a longitudinal external magnetic field. In terms of $Q^{a t b t^{\prime}}$ the susceptibility is given by

$$
\chi=\frac{\beta}{M^{2}} \sum_{a, b=1}^{n} \sum_{t, t^{\prime}=0}^{M-1} Q^{a t b t^{\prime}} .
$$

In Eqs. (20) and (22) the right-hand-sides should be evaluated at the saddle-point values.

The matrix elements $Q^{a t b t^{\prime}}$ are the order parameters of the model,

$$
\left.Q^{a t b t^{\prime}}=\frac{1}{N} \sum_{i=1} \overline{\left\langle\sigma_{i}^{a t} \sigma_{i}^{b t^{\prime}}\right.}\right\rangle
$$

Because of the translational invariance in the Trotter time direction the diagonal terms in the replica indices depend on the time-difference only,

$$
Q^{a t a t^{\prime}}=q_{d}\left(t-t^{\prime}\right)
$$


Notice that due to the periodic boundary condition $q_{d}(t)=q_{d}(M-t)$. In addition, as $q_{d}(0)=1$, only $\left(t-t^{\prime}\right)=1,2, \cdots$, int $\frac{M}{2}$ need to be considered. The off-diagonal elements in the replica indices, $Q^{a t b t^{\prime}}$ with $a \neq b$, are $t$ and $t^{\prime}$ independent as shown by Bray and Moore [24].

In order to determine the different phases of the model, we consider the following Ansätze:

Paramagnetic Phase

The matrices $Q$ and $\Lambda$ are taken to be diagonal in replica space

$$
Q^{a t b t^{\prime}}=q_{d}\left(t-t^{\prime}\right) \delta^{a b}, \quad \Lambda^{a t b t^{\prime}}=\lambda_{d}\left(t-t^{\prime}\right) \delta^{a b} .
$$

Using Eqs. (16), (17) and (18) the disorder-averaged free-energy per spin can be expressed as

$$
\beta \bar{f}=\frac{\beta^{2} J^{2}}{4 M^{2}}(p-1) \sum_{t \neq t^{\prime}}^{M-1} q_{d}^{p}\left(t-t^{\prime}\right)-\frac{\beta^{2} J^{2}}{4 M}+\sum_{t \neq t^{\prime}}^{M-1} \mathcal{C}_{\left(t-t^{\prime}\right)}-\ln \operatorname{Tr}_{\left\{\sigma^{t}\right\}} e^{H_{e f f}^{p m}}
$$

with

$$
H_{e f f}^{p m}=\frac{1}{M^{2}} \sum_{t \neq t^{\prime}}^{M-1} \lambda_{d}\left(t-t^{\prime}\right) \sigma^{t} \sigma^{t^{\prime}}+\sum_{t=0}^{M-1}\left(A+B \sigma^{t} \sigma^{(t+1)}\right) .
$$

Here, $q_{d}\left(t-t^{\prime}\right)$ and $\lambda_{d}\left(t-t^{\prime}\right)$ are obtained self-consistently from the extremum condition by summing over all $2^{M}$ spin configurations $\sigma^{t}= \pm 1$ :

$$
\begin{aligned}
q_{d}\left(t-t^{\prime}\right) & =\frac{\operatorname{Tr}_{\left\{\sigma^{t}\right\}}\left[e^{H_{e f f}^{p m}} \sigma^{t} \sigma^{t^{\prime}}\right]}{\operatorname{Tr}_{\left\{\sigma^{t}\right\}} e^{H_{\text {eff }}^{p m}}}, \\
\lambda_{d}\left(t-t^{\prime}\right) & =\frac{\beta^{2} J^{2} p}{4} q_{d}^{p-1}\left(t-t^{\prime}\right)+M^{2} \mathcal{C}_{\left(t-t^{\prime}\right)} .
\end{aligned}
$$

\section{Equilibrium spin-glass phase}

In order to characterize this phase we use a one-step replica symmetry breaking (RSB) Ansatz,

$$
\begin{aligned}
Q^{a t b t^{\prime}} & =\left(q_{d}\left(t-t^{\prime}\right)-q_{e a}\right) \delta^{a b}+q_{e a} \epsilon^{a b} \\
\Lambda^{a t b t^{\prime}} & =\left(\lambda_{d}\left(t-t^{\prime}\right)-\lambda_{e a}\right) \delta^{a b}+\lambda_{e a} \epsilon^{a b}
\end{aligned}
$$


Using Eqs. (16), (17) and (18) the disordered-averaged free-energy density becomes

$$
\begin{aligned}
\beta \bar{f}= & \frac{\beta^{2} J^{2}}{4 M^{2}}(p-1) \sum_{t \neq t^{\prime}}^{M-1} q_{d}^{p}\left(t-t^{\prime}\right)+(m-1) \frac{\beta^{2} J^{2}}{4}(p-1) q_{e a}^{p}-\frac{\beta^{2} J^{2}}{4 M} \\
& -\frac{1}{m} \ln \int d \mathcal{X}\left(\operatorname{Tr}_{\left\{\sigma^{t}\right\}} e^{H_{\text {eff }}^{e s g}}\right)^{m}+\sum_{t \neq t^{\prime}}^{M-1} \mathcal{C}_{\left(t-t^{\prime}\right)}
\end{aligned}
$$

with

$$
\begin{aligned}
H_{e f f}^{e s g}= & \frac{1}{M^{2}} \sum_{t \neq t^{\prime}}^{M-1}\left(\lambda_{d}\left(t-t^{\prime}\right)-\lambda_{e a}\right) \sigma^{t} \sigma^{t^{\prime}}-\frac{\lambda_{e a}}{M}+\frac{\sqrt{2 \lambda_{e a}}}{M} x \sum_{t}^{M-1} \sigma^{t} \\
& +\sum_{t}^{M-1}\left(A+B \sigma^{t} \sigma^{(t+1)}\right)
\end{aligned}
$$

and the integration measure

$$
d \mathcal{X} \equiv \frac{d x}{\sqrt{2 \pi}} e^{-\frac{x^{2}}{2}}
$$

Here and in what follows all integrals over $x$ go from $-\infty$ to $\infty$.

As in the paramagnetic phase, the order parameters $q_{d}\left(t-t^{\prime}\right), q_{e a}, \lambda_{d}\left(t-t^{\prime}\right)$ and $\lambda_{e a}$ are determined self-consistently from the extremum conditions that involve a sum over all $2^{M}$ spin configurations, $\sigma^{t}= \pm 1$ :

$$
\begin{aligned}
q_{d}\left(t-t^{\prime}\right) & =\frac{\int d \mathcal{X}\left(\operatorname{Tr}_{\left\{\sigma^{t}\right\}} e^{H_{\text {eff }}^{e s g}}\right)^{m-1}\left(\operatorname{Tr}_{\left\{\sigma^{t}\right\}} e^{H_{\text {eff }}^{e s g}} \sigma^{t} \sigma^{t^{\prime}}\right)}{\int d \mathcal{X}\left(\operatorname{Tr}_{\left\{\sigma^{t}\right\}} e^{H_{e f f}^{e s g}}\right)^{m}}, \\
q_{e a} & =\frac{\int d \mathcal{X}\left(\operatorname{Tr}_{\left\{\sigma^{t}\right\}} e^{H_{e f f}^{e s g}}\right)^{m-2}\left(\operatorname{Tr}_{\left\{\sigma^{t}\right\}} e^{H_{e f f}^{e s g}} \sum_{t} \sigma^{t} / M\right)^{2}}{\int d \mathcal{X}\left(\operatorname{Tr}_{\left\{\sigma^{t}\right\}} e^{H_{e f f}^{e s g}}\right)^{m}}, \\
\lambda_{d}\left(t-t^{\prime}\right) & =\frac{\beta^{2} J^{2} p}{4} q_{d}^{p-1}\left(t-t^{\prime}\right)+M^{2} \mathcal{C}_{\left(t-t^{\prime}\right)}, \\
\lambda_{e a} & =\frac{\beta^{2} J^{2} p}{4} q_{e a}^{p-1} .
\end{aligned}
$$

As it has been discussed in a number of papers on classical [25] and quantum [15. 16. 17. 26] spin-glass models, two choices for the determination of the breakingpoint $m$ lead to different physical results. The use of the extremum condition, that corresponds to looking for the value of $m$ that renders the disorder-averaged free-energy stationary, leads to

$$
m=I^{-1}\left[m^{2} \frac{\beta^{2} J^{2} p}{4}(p-1) q_{e a}^{p}+\ln \int d \mathcal{X}\left(\operatorname{Tr}_{\left\{\sigma^{t}\right\}} e^{H_{e f f}^{e s g}}\right)^{m}\right]
$$


where

$$
I=\frac{\int d \mathcal{X}\left(\operatorname{Tr}_{\left\{\sigma^{t}\right\}} e^{H_{e f f}^{e s g}}\right)^{m} \ln \left(\operatorname{Tr}_{\left\{\sigma^{t}\right\}} e^{H_{\text {eff }}^{e s g}}\right)}{\int d \mathcal{X}\left(\operatorname{Tr}_{\left\{\sigma^{t}\right\}} e^{H_{e f f}^{e s g}}\right)^{m}} .
$$

With this choice one describes the equilibrium properties of the model.

This Ansatz yields the exact solution [17] to the spherical version of the $p \geq 3$ model. When $p=2$ the spherical model is solved by a simpler replica symmetric (RS) form. The stability of the one-step Ansatz for quantum $S U(2)$ models can be tested by extending the analysis of de Almeida and Thouless [28]. When the lowest eigenvalue, that is also called the replicon, vanishes, the one-step RSB Ansatz becomes marginally stable. When the replicon is negative, this Ansatz is unstable.

We do not expect the one-step RSB Ansatz to be stable everywhere in the phase diagram in the case of discrete spins. Indeed, by evaluating the replicon at the order parameters and break-point obtained from the extremum conditions we found that the one-step RSB Ansatz is unstable in the full spin-glass phase when $p=$ 2 [Sherrington-Kirkpatrick (SK)] model indicating the need to break the replica symmetry further. In the case of the $p \geq 3$ classical spin model, the one-step RSB Ansatz is unstable below a temperature $T_{g}<T_{s}$ as shown by Gardner [29] in the classical case. Thus, the solution for the classical Ising $p$ spin model also requires full RSB at very low temperatures. $T_{g}$ depends on the parameter $p$ and, as expected, it tends to $T_{s}=J$ when $p \rightarrow 2^{+}$and it vanishes when $p \rightarrow \infty$.

When quantum fluctuations are taken into account we thus expect to find a Gardner line of instability. A careful study of how this line depends on $\Gamma$ and the coupling to the bath requires to solve the quantum problem at rather low temperatures. This is done in Sect. 3 where we compute the location of the Gardner instability line. As seen in Fig. [7 the region where the one-step RSB static Ansatz is unstable is quite small. Outside this region the one-step RSB Ansatz is exact and can be studied to study the properties of the $p \geq 3$ quantum $S U(2)$ model. Elsewhere, and for $p=2$, we shall regard this solution as a suitable approximation to the correct solution.

\section{$\underline{\text { Dynamic spin-glass phase }}$}

The marginality condition leads to a different equation for $m$. With this condition one requires that the saddle-point is only marginally stable, i.e. the matrix

of quadratic fluctuations has a zero replicon eigenvalue (and one does not impose the condition of extreme on $m$ ). It has been checked by comparison to the real- 
time dynamics 9], that this condition yields the freezing transition of the spherical quantum $p$-spin model with $p \geq 3$ coupled to the oscillator reservoir at the initial time $t=0$ [10]. Here we use it as an indication of where such a dynamic transition line should be located for the $S U(2)$ quantum spin systems.

Adapting the calculation of de Almeida and Thouless [28] to the quantum problem under study we find that the replicon eigenvalue is given by

$$
\lambda_{R}=P-2 Q+R
$$

with

$$
\begin{aligned}
P & =1-k q_{e a}^{p-2} t \\
Q & =-k q_{e a}^{p-2} u \\
R & =-k q_{e a}^{p-2} r .
\end{aligned}
$$

The factors $r, u$ and $t$ are

$$
\begin{aligned}
r & =\left\langle\sigma^{a} \sigma^{b} \sigma^{c} \sigma^{d}\right\rangle=\frac{\int d \mathcal{X}\left(\operatorname{Tr}_{\sigma^{t}} e^{H_{\text {eff }}^{d s g}}\right)^{m-4}\left(\operatorname{Tr}_{\sigma^{t}} e^{H_{\text {eff }}^{d s g}}\left(\sum_{t} \sigma^{t} / M\right)\right)^{4}}{\int d \mathcal{X}\left(\operatorname{Tr}_{\sigma^{t}} e^{H_{\text {eff }}^{d s g}}\right)^{m}} \\
u & =\frac{1}{M^{2}} \sum_{t \tau}\left\langle\sigma^{a t} \sigma^{b} \sigma^{a \tau} \sigma^{d}\right\rangle \\
& =\frac{\int d \mathcal{X}\left(\operatorname{Tr}_{\sigma^{t}} e^{H_{\text {eff }}^{d s g}}\right)^{m-3}\left(\operatorname{Tr}_{\sigma^{t}} e^{H_{\text {eff }}^{d s g}}\left(\sum_{t} \sigma^{t} / M\right)\right)^{2} \operatorname{Tr}_{\sigma^{t}} e^{H_{\text {eff }}^{d s g}}\left(\sum_{t t^{\prime}} \sigma^{t} \sigma^{t^{\prime}} / M^{2}\right)}{\int d \mathcal{X}\left(\operatorname{Tr}_{\sigma^{t}} e^{H_{\text {eff }}^{d s g}}\right)^{m}} \\
t & =\frac{1}{M^{4}} \sum_{t t^{\prime} \tau \tau^{\prime}}\left\langle\sigma^{a t} \sigma^{b t^{\prime}} \sigma^{a \tau} \sigma^{b \tau^{\prime}}\right\rangle \\
& =\frac{\int d \mathcal{X}\left(\operatorname{Tr}_{\sigma^{t}} e^{H_{\text {eff }}^{d s g}}\right)^{m-2}\left(\operatorname{Tr}_{\sigma^{t}} e^{H_{\text {eff }}^{d s g}}\left(\sum_{t t^{\prime}} \sigma^{t} \sigma^{t^{\prime}} / M^{2}\right)\right)^{2}}{\int d \mathcal{X}\left(\operatorname{Tr}_{\sigma^{t}} e^{H_{\text {eff }}^{d s g}}\right)^{m}}
\end{aligned}
$$

Here, we have defined

$$
k \equiv \frac{\beta^{2} J^{2}}{2} p(p-1)
$$

and $H_{e f f}^{d s g}$ is the Hamiltonian of by Eq. (33). Finally, the values of $q_{d}\left(t-t^{\prime}\right)$ and $q_{e a}$ are fixed by the extremal conditions. 


\section{Results}

In this Section we describe the outcome of solving the equations we derived in the previous Section and we discuss how the coupling to the Ohmic bath of harmonic oscillators modifies the behavior of the spin model.

\subsection{Numerical method}

The free-energy density and derived magnitudes depend on the parameter $M$ that in practice takes finite values. Several strategies were proposed to study the limit $M \rightarrow \infty$. Usadel and Schmitz [30] noted that $M$ should be such that $\beta \Gamma / M \ll 1$. For low temperatures this criterium becomes quickly impractical since one cannot perform the complete sum over states for such large values of $M$. As an alternative, these authors proposed to use a Montecarlo procedure to estimate the sum over configurations when $M$ is large [30, 31].

In this paper we use another method that has been previously used to study the isolated quantum SK [19] and the $S U(2) p$-spin [15] models in a transverse field. We compute each magnitude using a direct spin sumation (DSs) with $M$ ranging form $M=8$ to $M=13$. The six values obtained are the input for a polynomial extrapolation which gives the $M \rightarrow \infty$ limit for the considered magnitud. For almost all cases, we extrapolate using the $(1 / M)^{2}$ law 32. Special care is taken for the study of the function $q_{d}(\tau)$ that is known on the imaginary-time grid $0, \frac{\beta \hbar}{M}, \frac{2 \beta \hbar}{M}, \cdots, \beta \hbar$. We interpolate these sets of points with splines for each value of $M$. As the value of $M$ increases the density of points also increases and we have a better description of the curve. Finally we use the result of the function $q_{d}^{M}(\tau)$ at $\tau$ as the input for the polynomial extrapolation. Because of the additional difficulties introduced by the functional nature of $q_{d}(\tau)$ we considered a linear extrapolating scheme in this case.

Before discussing the behavior of the model in detail let us illustrate the dependence on the number of time slices $M$ used in the DSS numerical method. In Fig. 1 we show the diagonal order parameter $q_{d}(\tau)$ as a function of $\tau$ at constant temperature and transverse magnetic field for the $p=3$ model. Six values of $M$ are shown, $M=8, \cdots, 13$. In Fig. 2 we display the free-energy density of the different phases of the $p=3$ model with $\eta=1$. The four curves correspond to three values of $M, M=8,9,10$, and the result of the extrapolation to $M \rightarrow \infty$. In both cases the variation of the curves with $M$ is indeed very smooth.

Even if the extrapolating method of Goldschmidt and Lai 19 is simple to im- 

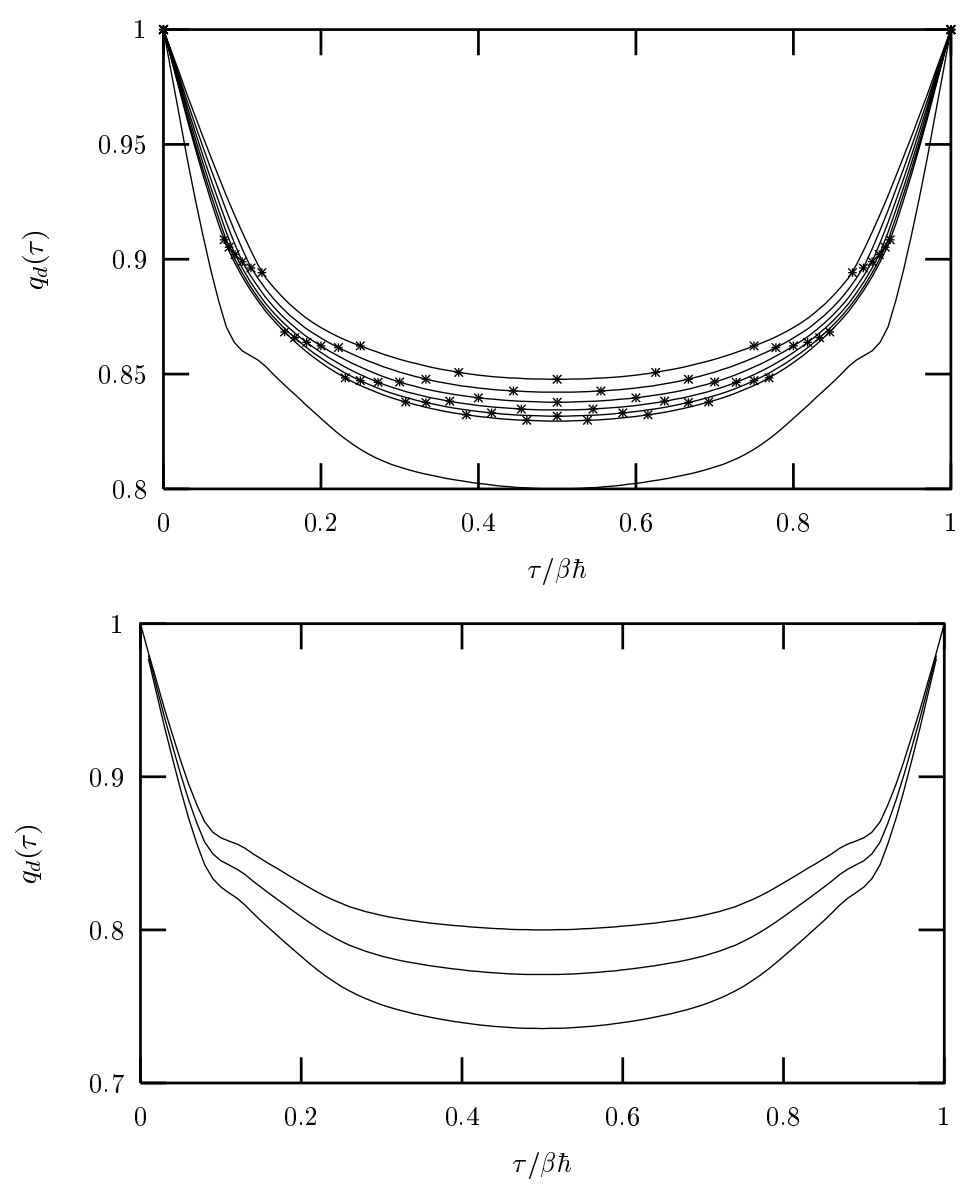

Figure 1: Upper panel: The diagonal imaginary-time dependent order parameter $q_{d}(\tau)$ in the $p=3$ model as a function of $\tau /(\beta \hbar)=t / M$ with $t$ the Trotter time, $t=$ $0,1, \cdots, M$. The temperature is $T=0.3$ and the transverse magnetic field is $\Gamma=0.8$. The coupling to the bath is $\eta=1$. The solutions for $M=8,9,10,11,12,13$ are shown from top to bottom, (with linepoints, the points being the numerical data and the lines representing the result of splines) and the lowest curve (thin line) is the result of the extrapolation to $M \rightarrow \infty$. Lower panel: The limiting curve $\lim _{M \rightarrow \infty} q_{d}^{M}(\tau)$ for three couplings to the environment: $\eta=0$ (bottom), $\eta=0.5$ (middle) and $\eta=1$ (top). The other parameters are the same as in the upper panel. (See the text for the details of the extrapolated method used.) 


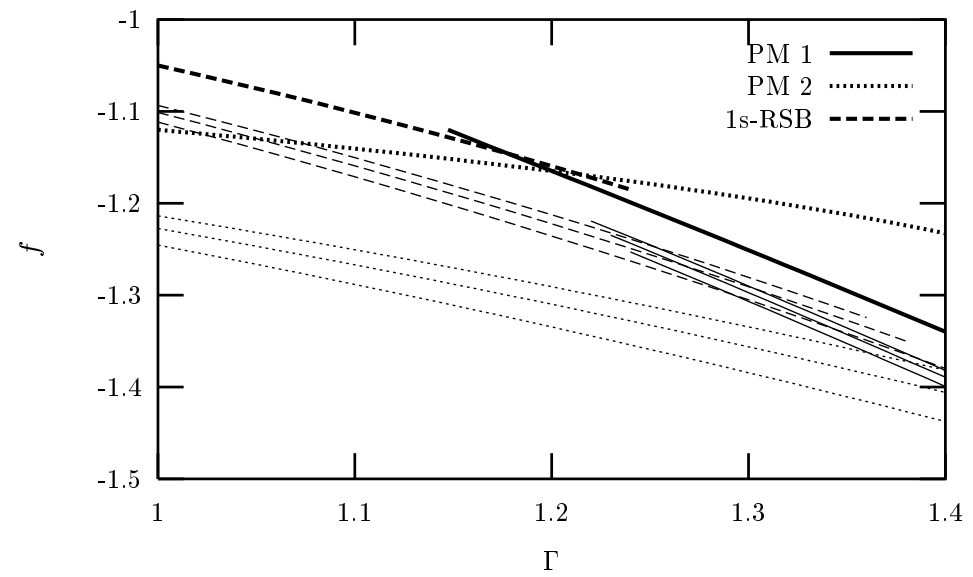

Figure 2: Disorder-averaged free-energy density, $\bar{f}$, of the quantum $S U(2) p=3$ model at temperature $T=0.3$ as a function of the transverse magnetic field $\Gamma$. The coupling to the bath is $\eta=1.0$. The three phases of the model are represented: a physical paramagnet labelled PM1, an unphysical paramagnet that one discard on physical grounds labeled PM2 and the spin-glass. The values of $\bar{f}$ obtained for finite $M$ are shown with thin lines $[M=8$ (bottom), $M=9$ (middle) and $M=10$ (top)]. The result of the extrapolation to $M \rightarrow \infty$ is displayed with bold lines.

plement and very efficient, it is also limited when the temperature decreases. The extrapolation is less clear at lower temperatures.

\subsection{Static phases}

Let us first discuss the effect of the environment on the static phase diagram of the $p=2$ (SK) and $p \geq 3$ quantum $S U(2)$ spin models. The spin-glass disorderaveraged free-energy densities are obtained using the one-step RSB Ansatz discussed in Sect. 2. We discuss the limits of validity of this Ansatz below.

As in other disordered quantum spin models [15, 16, 17, 22] two paramagnetic solutions coexist. As in the spherical $p$-spin model coupled to a bath the one labeled PM2 in Fig. 2 can be discarded since its entropy becomes negative at sufficiently low temperatures. Thus, we do not discuss it further in this paper.

The critical line $\left(T_{s}, \Gamma_{s}\right)$ separating the paramagnetic (PM) and spin-glass (SG) phases is determined by the values of the pairs $(T, \Gamma)$ where the physical paramagnetic (called PM1 in Fig. 22) and spin-glass free-energy densities cross.

We show in the upper and lower panels of Fig. 3 the static critical line in the 
$(T, \Gamma)$ plane separating a high $T$, high $\Gamma$ paramagnetic phase (PM) from a low $T$, low $\Gamma$ spin-glass (SG) for the $p=2$ and $p=3$ models, respectively. The three curves in each figure correspond to $(\eta=0)$ and two non zero couplings $(\eta=0.5,1)$ from bottom to top.

For both models, the classical transition temperature, $T_{s}^{c l a s s}$, corresponding to $\Gamma_{s} \rightarrow 0$, remains unchanged by the coupling to the quantum heat reservoir. This value is $T_{s}=J$ for when $p=2[23$ ] and it coincides with the one given by Gross and Mézard, $T_{s} \approx 0.67$, for the classical problem with $p=3[33$.

For the three values of $\eta$, the static critical transverse field, $\Gamma_{s}(T)$, is a decreasing function of $T$, which is consistent with the fact that quantum fluctuations tend to destroy the glassy phase. We also see from the figures that the coupling to a quantum thermal bath favors the formation of the glassy phase: the coupling to the environment effectively reduces the strength of the quantum fluctuations that tend to destroy it. For any value of the temperature that satisfies $T<T_{s}^{\text {class }}$ the extent of the spin-glass phase is larger for stronger couplings to the bath. Moreover, we observe that the effect of the bath is stronger for lower temperatures.

When $p=2$ the transition is always continuous and second-order thermodynamically. For $p=3$ instead, as in the spherical case [16, 17, 10] and the isolated quantum $S U(2)$ model [15], an interesting change from a second-order to a firstorder transition appears. We demonstrate these statements by displaying in Figs. 4 and [5] the behavior of the free-energy density, entropy and susceptibility of the $p=3 S U(2)$ spin model as a function of the transverse field for $T=0.5>T_{s}^{*}$ and $T=0.3<T_{s}^{*}$.

At sufficiently high temperatures, $T \geq T_{s}^{*}$, one finds a spin-glass solution for increasing transverse fields until the break-point $m$ reaches the value $m=1$. The values $(T, \Gamma)$ where $m=1$ coincides with the ones obtained by analyzing the crossing of the free-energy densities of the paramagnetic and spin-glass solutions. Thus, for the chosen temperature $T \geq T_{s}^{*}$ this is the critical transverse field. Even if the Edwards-Anderson parameter, $q_{e a}$, and the diagonal element, $q_{d}(\tau)$, are non-zero at this point in parameter space, one can check, as shown in Fig. [4 that the entropy and susceptibility do not show a jump. Thus, for $T \geq T_{s}^{*}$ the transition is discontinuous [due to the jump in $q_{e a}$ and $q_{d}(\tau)$ ] but of second order thermodynamically.

The situation is different at lower temperatures. In Fig. 5 we show the free energy, entropy and susceptibility of the $p=3 S U(2)$ model for $T=0.3<T_{s}^{*}$. In this case, the point in which the free-energy of the paramagnetic and spin-glass solution 

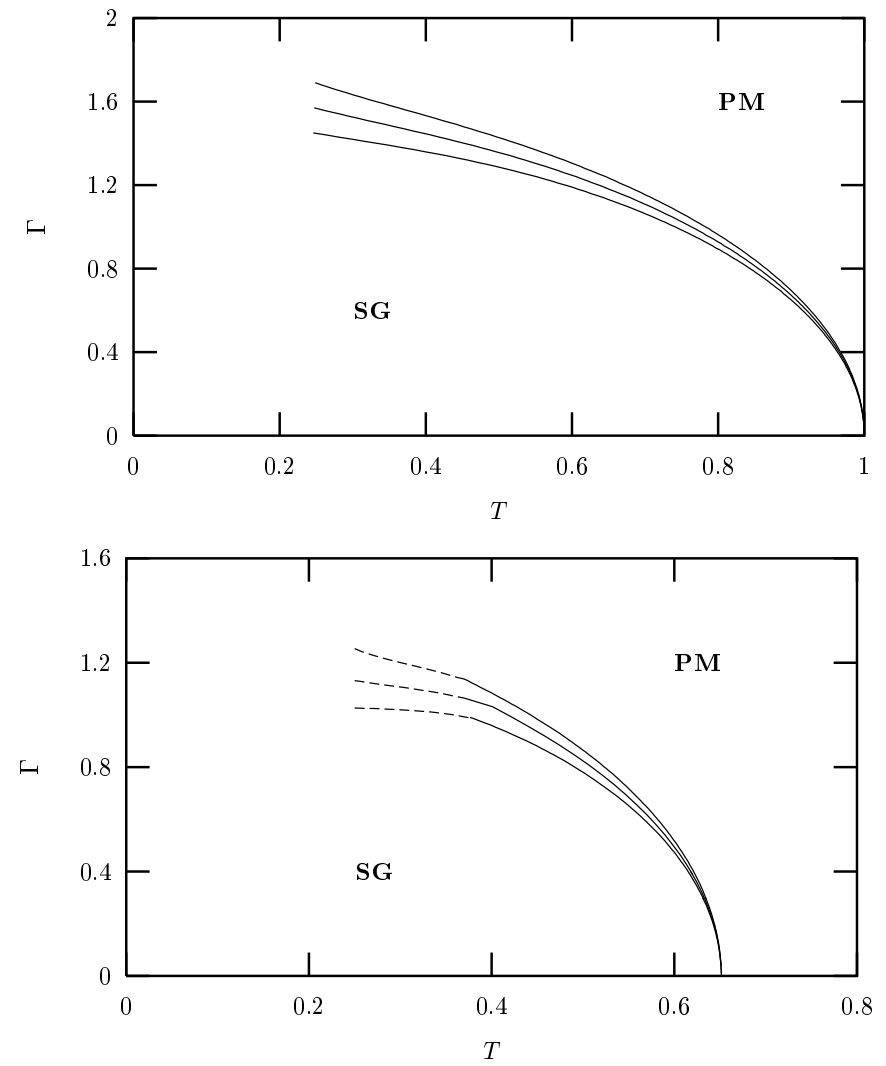

Figure 3: Upper panel: Static phase diagram for the $p=2$ model as obtained using the DSs technique for finite number of time slices $M$ and extrapolating the data to $M \rightarrow \infty$. The three lines correspond to $\eta=0,0.5,1$, from bottom to top. Lower panel: Static phase diagram for the $p=3$ model obtained using the same numerical method. The continuous line (dashed line) indicates a second order (first order) phase transition. The critical lines continue below the lowest value of $T$ for which we trust the algorithm, $T \approx 0.25$, to reach a quantum critical point at $T=0$. 

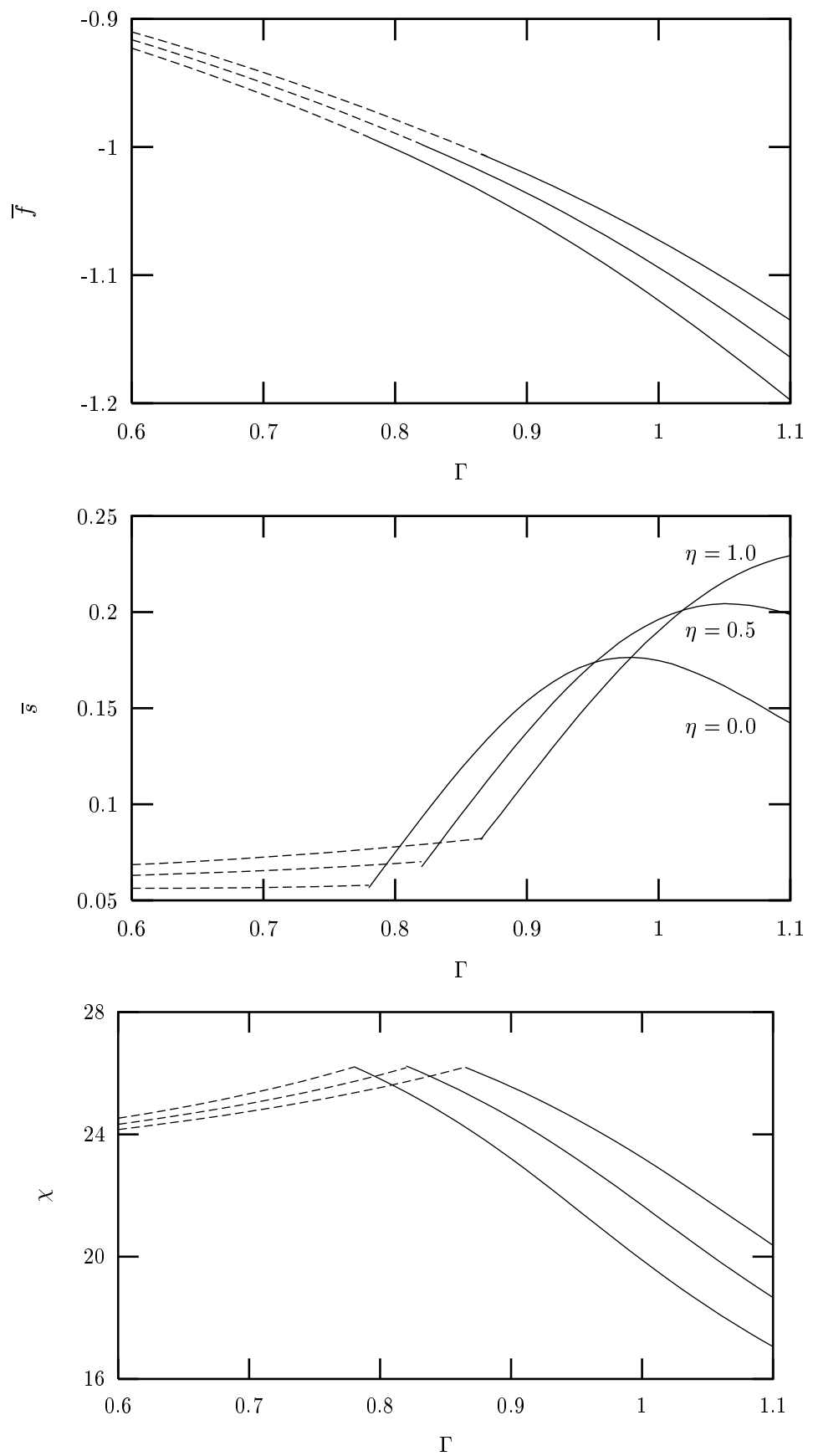

Figure 4: Free energy density, $\bar{f}$, entropy, $\bar{s}$, and susceptibility, $\chi$, as functions of the transverse field, $\Gamma$, for the $p=3$ model at $T=0.5>T_{s}^{*}$ for three values of the coupling to the bath, $\eta=0,0.5,1$. The continuous (dashed) line corresponds to the paramagnetic (glassy) phase. The entropy and susceptibility are continuous at the transition indicating a second order phase transition 
cross corresponds to $m<1$ and as shown in the figure this leads to a discontinuity of the entropy and susceptibility. In this case, the transition is discontinuous and first-order thermodynamically.

In Fig. 6] we show the dependence of $q_{e a}$ and $m$ on the critical temperature $T_{s}$ for three values of the coupling to the bath. The model is again the $p=3$ quantum $S U(2)$ spin-glass. As already mentioned we observe that for all temperatures $q_{e a}$ is different from zero, leading to a discontinuous phase transition. $m$ equals one for $T_{s} \geq T_{s}^{*}$ but $m<1$ for $T_{s} \leq T_{s}^{*}$. The figure also shows that $T_{s}^{*}$ decreases with increasing coupling to the bath $\eta$. Again, this result is reminiscent of what found in the spherical case [10.

\subsection{Stability of the one-step static solution}

In order to study the stability of the one-step solution we evaluated the replicon eigenvalue $\lambda_{R}$ on the values of the order parameters and $m$ obtained from the static solution, and we searched for the parameters $(T, \Gamma)$ such that $\lambda_{R}$ vanishes. In the classical limit this yields Gardner's classical critical temperature that takes a rather low value, $T_{G}(\Gamma=0) \approx 0.25[29$. Since we expect to find a decreasing value of the instability temperature with the strength of the transverse field, we need to control the numerical algorithm for $T<0.25$. Even if this might seem, at first sight, impossible, we managed to obtain sensible results keeping reachable values of $M, M \leq 13$, since the small values of the transverse field compensate the large value of $\beta$ in the condition $\beta \Gamma / M \ll 1$.

First, we analyzed the $p=2$ case that corresponds to the SK model in a tranverse field. In the absence of the environment we found that the one-step RSB solution is not stable in the full spin-glass phase supporting the idea that the solution to the statics of this model needs a full RSB scheme, just as in its classical limit and in contrast to recent claims in the literature [34].

In Fig. 7 we compare the static critical line $\left(T_{s}, \Gamma_{s}\right)$ as found from the one-step RSB Ansatz, with Gardner's line of instability for the $p=3$ model. We see that the region where the one-step RSB static Ansatz is not stable is quite small. Since we only trust the extrapolation from low values of $M$ to $M \rightarrow \infty$ above temperatures of the order of $T \approx 0.1$, we do not explicitly extrapolate the instability line to lower temperatures. Nevertheless, the existing data suggest that in the zero temperature limit the static critical transverse field, $\Gamma_{s}$, and Gardner's critical field, $\Gamma_{G}$, do not 

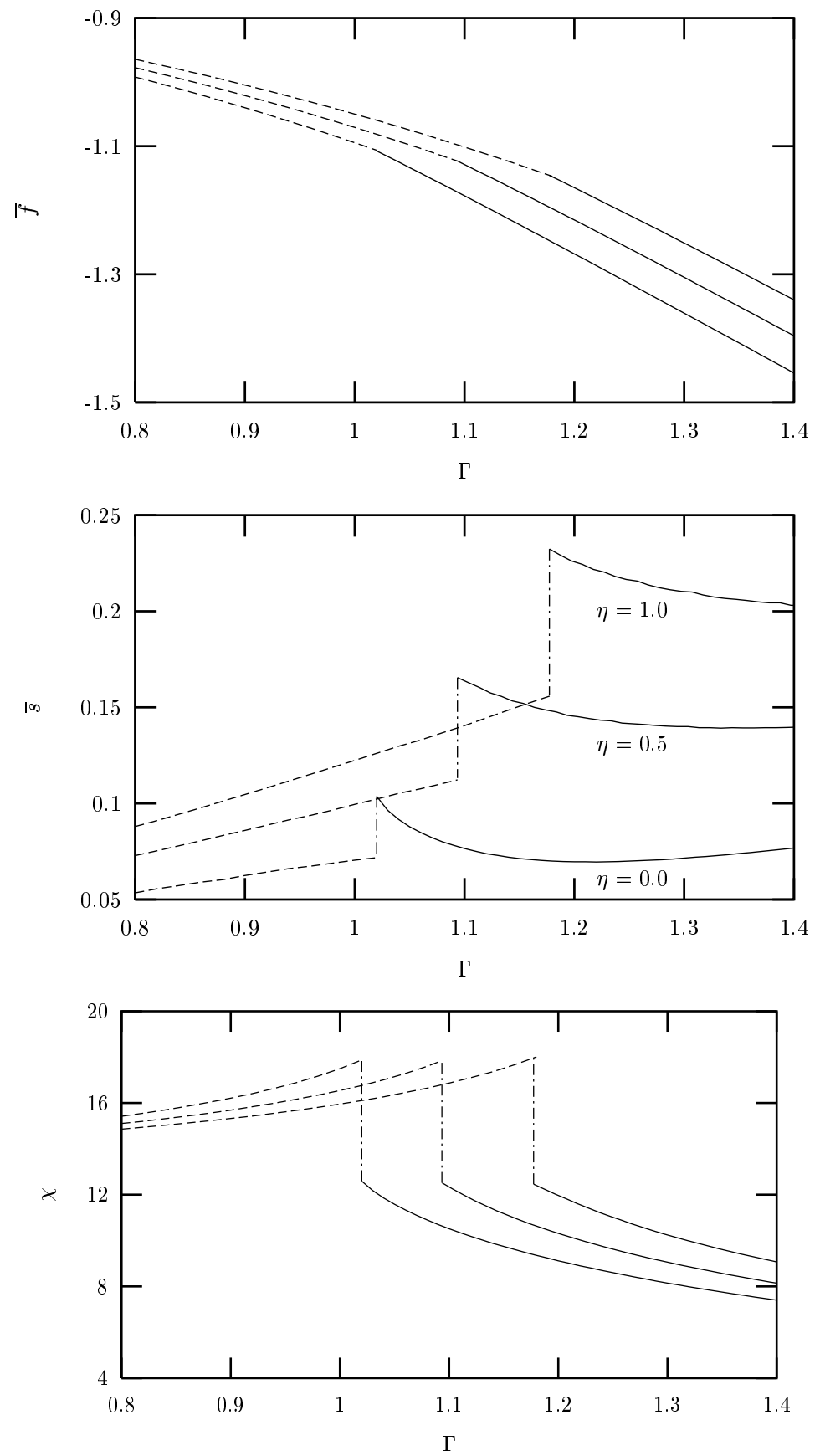

Figure 5: Free energy, $\bar{f}$, entropy, $\bar{s}$, and susceptibility, $\chi$, as function of the transverse field, $\Gamma$, for the $p=3$ model at $T=0.3<T_{s}^{*}$ for three values of the coupling to the bath, $\eta=0,0.5,1$. The continuous (dashed) line corresponds to the paramagnetic (glassy) phase. The entropy and susceptibility are discontinuous at the transition indicating a first order phase transition 


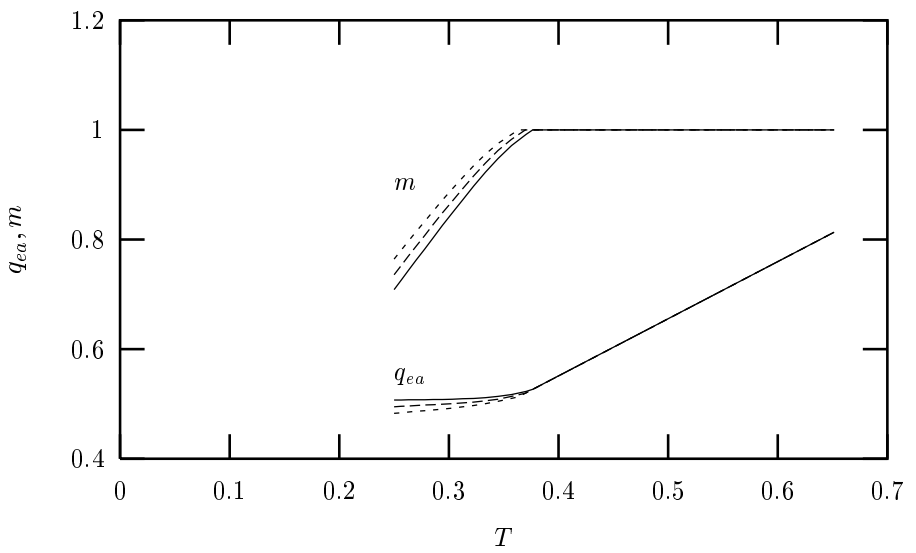

Figure 6: Dependence of $m$ and $q_{e a}$ on the critical temperature $T_{s}(\Gamma, \eta)$ for the $p=3$ model. Three values of $\eta$ are considered, $\eta=0,0.5,1$. For increasing values of $\eta$ the interval in which $m=1$ increases and, hence, the region where a thermodynamic first order transition occurs decreases.

coincide $\Gamma_{s}\left(T_{s}=0\right)>\Gamma_{G}\left(T_{G}=0\right)$.

\subsection{The dynamic transition}

As already explained in Sect. 2 the value of $m$ found by setting the replicon eigenvalue to zero leads to different equations that encode some information about the non-equilibrium relaxation dynamics of the system. Using this prescription we obtained, for the $p \geq 3$ models a different critical line that lies above the static transition. This result is similar to those found in a series of other classical [25] and quantum [15, 16, 17] problems. In Fig. 8] we compare the static and marginal critical lines for the $p=10$ quantum $S U(2)$ model. We chose a larger value of $p$ to make the difference between the two lines easier to visualize. The glassy static region is smaller than the glassy region determined by the marginality condition. When approaching the glassy phase from any direction in parameter space, the dynamic transition, associated to the line of marginal stability, occurs before the static one. As on the critical static line, the curve determined with the marginal stability criterion is made of two pieces, on one of them the transition is of secondorder (indicated with a solid line on Fig. 8) and on the other the transition is of first-order (indicated with a dashed line on the same figure). The first-order nature of the dynamic transition is displayed by, for instance, a jump in the asymptotic 


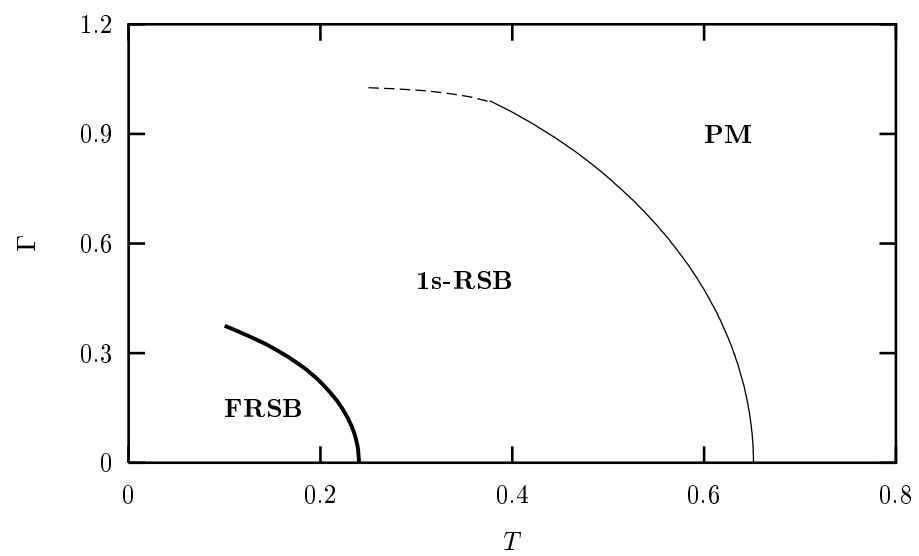

Figure 7: Comparison between the static critical line $\left(T_{s}, \Gamma_{s}\right)$ and Gardner's instability line $\left(T_{G}, \Gamma_{G}\right)$ for the $p=3$ model with $\eta=0$.

value of the averaged internal energy. The marginal tricritical point occurs at higher temperature than the static one.

The external noise also has a strong effect on the dynamic critical line. The stronger the coupling to the environment (larger value of $\eta$ ), the larger the spinglass region in the phase diagram. This is also shown in Fig. 8 where a couple of curves, corresponding to $\eta=0$ and $\eta=0.5$ are drawn (see the caption in the figure for more details).

Finally, let us mention that there is an empirical relation between the value of the parameter $m$ as found from the marginality condition and how the fluctuationdissipation theorem is modified in the real-time non-equilibrium relaxation of the quantum model 9 , 10. Using this relation and interpreting then the parameter $m / T$ as an effective temperature [35] we find that the modification of the fluctuationdissipation theorem, and hence $T_{\text {eff }}$, depend on the strength of the coupling to the bath.

\section{Conclusions}

In this paper we studied the effect of an Ohmic quantum bath on the statics and dynamics of quantum disordered $S U(2)$ spin models of mean-field type. We found that the coupling to the enviromemnt favors the appearance of the spin-glass phase 


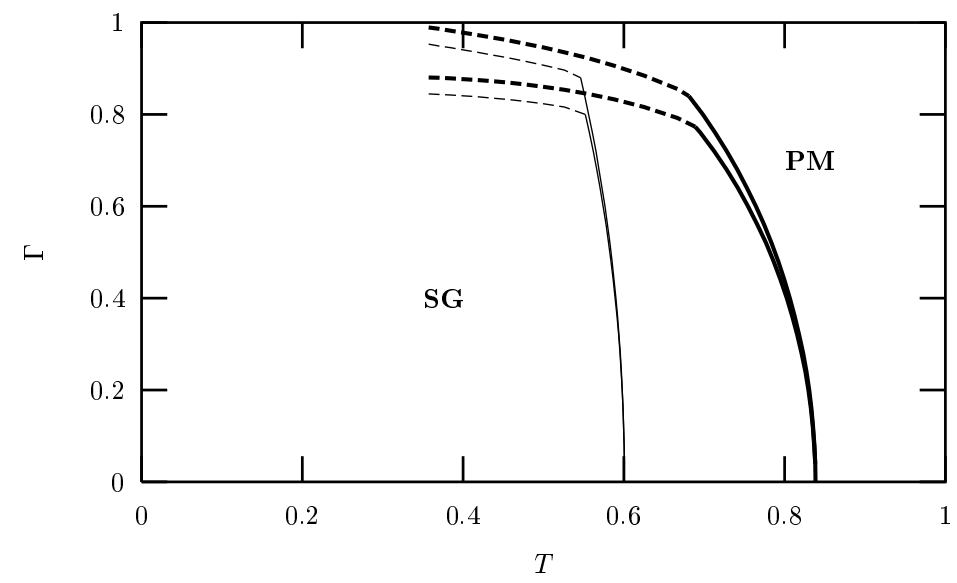

Figure 8: Comparison between the static and marginal critical lines for the $p=10$ model. The solid lines represent second order transitions and the dashed lines first order ones. The set of curves with $T_{s}^{\text {class }} \approx 0.6$ (thin lines) are the static transition lines for $\eta=0$ (below) and $\eta=1$ (above); the set of curves with $T_{s}^{\text {class }} \approx 0.82$ (bold lines) correspond to the dynamic transition for $\eta=0$ (below) and $\eta=1$ (above).

reducing the strength of the quantum fluctuations that tend to destabilize it. As in the case of the spherical model [10, 11] the phase transition is always second order for $p=2$. For $p \geq 3$ there exists a tricritial temperature $T^{\star}$ below which quantum fluctuations drive the transition first order. $T^{\star}$ decreases with the strength of the coupling to the bath. For $p \geq 3$ a dynamic precedes the equilibrium phase transition. The coupling to the bath also stabilizes the dynamic glassy phase.

It would be interesting to check if the same tendency to ordering appears in macroscopic spin models in finite dimensions. One could attempt to study this problem in the context of frustrated spin magnets or the much studied, numerically and analytically, quantum $S U(2)$ spin chain with and without disorder.

This problem is of interest for possible implementations of quantum computers where the interaction of the system with its environment needs to be controlled. The effect of an environment on the properties of Griffith phases has also been the focus of a hot debate [36]. We expect to report on these problems in the future. 
Acknowledegments We acknowledge financial support from the an Ecos-Sud travel grant, the ACI project "Optimisation algorithms and quantum disordered systems". LFC is research associate at ICTP Trieste and acknowledges financial support from the J. S. Guggenheim Foundation. G. S. L. is supported by EPSRC grants GR/N19359 and GR/R70309. This research was supported in part by the National Science Foundation under Grant No. PHYS99-07949. We thank F. Ritort for very useful discussions.

\section{References}

[1] A. J. Leggett, S. Chakravarty, A. T. Dorsey, M. P. A. Fisher, A. Garg and W. Zwerger, Rev. Mod. Phys. 59, 1 (1987); 67, 725 (1995).

[2] U. Weiss in Series Modern Condensed Matter Physics (World Scientific, Sigapore, 1993) Vol. 2.

[3] W. Wu, B. Ellmann, T. F. Rosenbaum, G. Aeppli, and D. H. Reich, Phys. Rev. Lett. 672076 (1991); W. Wu, D. Bitko, T. F. Rosenbaum, and G. Aeppli, Phys. Rev. Lett. 711919 (1993).

[4] R. Vollmer, T. Pietrus, H. v. Löhneysen, R. Chau, and M. B. Maple, Phys. Rev. B 61, 1218 (2000).

[5] Y. Tabata, D. R. Grempel, M. Ocio, T. Taniguchi, and Y. Miyako, Phys. Rev. Lett. 86, 524 (2001).

[6] F. Ladieu, J. Le Cochec, P. Pari, P. Trouslard, and P. Ailloud, Phys. Rev. Lett. 90, 205501 (2003). S. Ludwig and D. D. Osheroff, Phys. Rev. Lett. 91, 105501 (2003). S. Rogge, D. Natelson, and D. D. Osheroff, Phys. Rev. Lett. 76, 3136 (1996).

[7] D. R. Grempel, M.J. Rozenberg, Phys. Rev. Lett. 79, 389 (1998). M.J. Rozenberg and D. R. Grempel Phys. Rev. Lett. 81, 2550 (1998).

[8] H. Rieger and A. P. Young, Quantum spin glasses (Springer-Verlag, Berlin, 1996), cond-mat/9607005. R. Bhatt, Quantum spin glasses in "Spin-glasses and random fields", A. P. Young ed. (World Scientific, Singapore, 1997).

[9] L. F. Cugliandolo, and G. Lozano, Phys. Rev. Lett. 80, 4979 (1998), Phys. Rev. B 59, 915 (1999). 
[10] L. F. Cugliandolo, D. R. Grempel, G. Lozano, H. Lozza, and C. A. da Silva Santos, Phys. Rev. B 66, 014444 (2002).

[11] M. Rokhni, and P. Chandra, cond-mat/0301166.

[12] G. Biroli and O. Parcollet, Phys. Rev. B 65, 094414 (2002).

[13] N. Pottier, and A. Mauger, Physica A 28277 (2000).

[14] D. R. Grempel and M. J. Rozenberg, Phys. Rev. B 60, 4702 (1999).

[15] T. M. Nieuewenhuizen and F. Ritort, Physica A 250, 89 (1998).

[16] L. F. Cugliandolo, D. R. Grempel, and C. A. da Silva Santos, Phys. Rev. Lett. 85, 2589 (2000).

[17] L. F. Cugliandolo, D. R. Grempel, and C. A. da Silva Santos, Phys. Rev. B 64, 014403 (2001).

[18] G. Biroli, and L. F. Cugliandolo, Phys. Rev. B 64, 014206 (2001).

[19] Y. Y. Goldschmidt and P-Y Lai, Phys. Rev. Lett. 64, 2467 (1990). P-Y Lai and Y. Y. Goldschmidt, Europhys. Lett. 13, 289 (1990).

[20] R. P. Feynman and Vernon, Jr. Ann. Phys. (NY), 24, 118 (1963); R. P. Feynman, Statistical mechanics. Addison Wesley, 1972.

[21] A. Caldeira and A. Leggett, Phys. Rev. Lett. 46, 211 A. Caldeira and A. Leggett, Ann Phys. 149, 374 (1983).

[22] D. R. Grempel and M. J. Rozenberg, Phys. Rev. B 60, 4702 (1999).

[23] M. Mézard, G. Parisi and M. A. Virasoro, Spin glass theory and beyond (World Scientific, Singapore, 1987).

[24] A. J. Bray and M. A. Moore, J. Phys. C 13, L655 (1980).

[25] T. R. Kirkpatrick and D. Thirumalai, Phys. Rev. B 36, 5388 (1987). T. R. Kirkpatrick and P. G. Wolynes, Phys. Rev. B 36, 8552 (1987). L. F. Cugliandolo and J. Kurchan, Phys. Rev. Lett. 71, 173 (1993).

[26] T. Giamarchi and P. Le Doussal, Phys. Rev. B 5315206 (1996). A. Georges, O. Parcollet and S. Sachdev, Phys. Rev. Lett., 85, 840 (2000), Phys. Rev. B 63, 134406 (2001). G. Schehr, T. Giamarchi and P. Le Doussal, cond-mat/0212300.

[27] A. J. Bray and M. A. Moore, J. Phys C 12, L441 (1979).

[28] J. R. L. de Almeida and D. J. Thouless, J. Phys. A 11, 983 (1978). 
[29] E. Gardner, Nucl. Phys. B 257 [FS14], 747 (1985).

[30] K. D. Usadel and B. Schmitz, Solid State Comm. 64, 975 (1987).

[31] J. V. Alvarez and F. Ritort, J. Phys. A: Math. Gen. 29, 7355 (1996).

[32] M. Suzuki, Prog. Theor. Phys., 56,1454 (1976)

[33] D. J. Gross and M. Mézard, Nucl. Phys. B 240 [FS12], 431 (1984).

[34] D-H Kim and J-J Kim, Phys. Rev. B 66, 054432 (2002).

[35] L. F. Cugliandolo, J. Kurchan and L. Peliti, Phys. Rev. E 55, 3898 (1997).

[36] A. H. Castro-Neto and B. A. Jones Phys. Rev. B 62, 14975 (2000). A. J. Millis, D. Morr and J. Schmalian, Phys. Rev. B 66, 174433 (2002). 